\title{
Ability of the Schatzker classification to predict posteromedial fragmentation in tibial plateau fractures
}

\author{
Niemoller HG${ }^{1}$, Greyling P², Birkholtz F³, Goller R², Suleman F4, Postma TC \\ 1 BSc(UV), MBBCh(Wits); Orthopaedic surgeon in training, Kalafong Hospital, Pretoria \\ 2 MBChB(Pret), MMed(Orth)(Pret), FCS(Orth)(SA); Orthopaedic Surgeon, Steve Biko Academic Hospital, Pretoria \\ ${ }^{3}$ MBChB(Pret), MMed(Orth)(Pret), FCS(Orth)(SA); Orthopaedic Surgeon, Unitas Hospital, Walk-a-mile Centre of Excellence \\ ${ }^{4}$ MBChB(Pret), MMed(Radiology)(Pret), FCS(Rad)(SA); Radiologist, Professor, Steve Biko Academic Hospital, Pretoria Postma TC6 \\ MChD, DHSM, PhD; Associate Professor and Head of Clinical Unit and Statistician. Orthopaedic Surgery, Department of Orthopaedic Surgery, Steve Biko \\ Hospital, University of Pretoria
}

Corresponding author: Dr H Niemoller, Second Avenue, Menlopark, Pretoria; email: heinrichniemoller@gmail.com

\begin{abstract}
Background: The Schatzker classification is the most widely accepted system used to classify tibial plateau fractures. The presence of posteromedial fragments in the more severe fracture types is known, but the presence of posteromedial fragmentation in the less severe fracture types is unknown. The ability of the Schatzker classification to predict posteromedial fragmentation was evaluated.

Methods: Two hundred patients were reviewed of which only 67 met the inclusion criteria. The X-rays were reviewed by three independent orthopaedic surgeons and classified according to the Schatzker classification. A radiologist reviewed the CT scans and noted the presence or absence of a posteromedial fragment and if present, the largest diameter of the fragment was measured.

Results: The mean age of the sample was 44.79 (SD: 14.03) years. Seventy-five per cent of the females ( $n=33$ ) presented with posteromedial fragmentation compared to $65.20 \%$ of the males $(n=44)$ (chi2-test, $P=0.399)$. The incidence of posteromedial fragmentation varied between 15.8 and $26.3 \%$ for Schatzker 1 and 2 fractures and 73.7 and $84.2 \%$ for Schatzker 3 to 6 (chi ${ }^{2}$-test, $p<0.001$ ) based on the three independent assessments. The mean length of the posteromedial fragmentation ranged from 41.87 to $47.77 \mathrm{~mm}$ for Schatzker 1 and 2 fractures, and 44.74 to $46.12 \mathrm{~mm}$ for Schatzker 3 to 6 for the three assessors (statistically not significant $[T$-test, $P=0.536$, $P=0.551$ and $P=0.652])$.

Conclusion: The Schatzker classification by itself is not adequate to identify all fractures with posteromedial fragmentation. There is a higher association of posteromedial fragmentation with fracture types 3 to 6 . There is a high probability of missing a significantly sized posteromedial fragment in Schatzker type 1 and 2 fractures if a CT scan is not performed which might influence and compromise fracture stability, joint congruency and the ability to rehabilitate optimally.
\end{abstract}

Level of evidence: Level 4

Key words: tibial plateau fracture, Schatzker classification, CT scan, posteromedial fragmentation

Citation: Niemoller HG, Greyling P, Birkholtz F, Goller R, Suleman F, Postma TC. Ability of the Schatzker classification to predict posteromedial fragmentation in tibial plateau fractures. SA Orthop J 2018;17(2):16-19. http://dx.doi.org/10.17159/2309-8309/2018/v17n2a2

Editor: Prof Anton Schepers, University of the Witwatersrand

Received: May $2017 \quad$ Accepted: October $2017 \quad$ Published: May 2018

Copyright: (c) 2018 Niemoller HG, et al. This is an open-access article distributed under the terms of the Creative Commons Attribution Licence, which permits unrestricted use, distribution and reproduction in any medium, provided the original author and source are credited.

Funding: This study did not require major funding. Minimal costs incurred for stationery were covered by the first author.

Conflict of interest: The authors have no conflicts of interests with respect to this article. 


\section{Introduction}

Tibial plateau fractures represent approximately $1.2 \%$ of all fractures and are therefore relatively uncommon. These fractures have a bimodal distribution: younger individuals with high-energy injuries and lower energy elderly patients who fall due to osteopaenia. ${ }^{1}$

Classifying the injury is important for pre-operative planning and prognosis. The range of tibial plateau injuries present across a full spectrum, including open or closed and displaced or non-displaced fractures. The more severe the comminution, the higher the chance of soft tissue compromise and neurovascular complications. ${ }^{2}$

The goals of treatment for intra-articular fractures are three-fold: the achievement of a stable fixation, the anatomical reduction of the joint surface and the preservation of a range of motion. ${ }^{3}$

The two most common systems that are used to classify fractures and dislocations are the AO/OTA system and the Schatzker system. Of these two systems, the Schatzker system is the most reliable and widely accepted classification system, internationally. ${ }^{3}$ Figure 1 highlights the important components of the Schatzker classification. ${ }^{3}$

Bicondylar tibial plateau fracture management remains therapeutically challenging. A study done by David et al. evaluated the frequency and morphology of the posteromedial fragment in this injury pattern. They concluded that the $\mathrm{AO}$ classification system missed $6 \%$ of posteromedial fragments on $\mathrm{X}$-rays. ${ }^{4}$

In an attempt to increase the sensitivity of these classification systems, Martijn et al. researched whether the addition of a computer tomography (CT) scan would increase inter- and intra-observer agreement or not. The study also aimed to evaluate inter-observer agreement for fracture classification according to the Schatzker classification as well as the treatment of tibial plateau fractures using $\mathrm{X}$-rays alone in comparison to using both X-rays and CT scans. They concluded that CT scans are not advised for all tibial plateau fractures. ${ }^{5}$

This, however, is in contrast with what Zhu et al. found when they compared the Schatzker classification (X-ray-based classification) with the three-column classification system (CT scan-based classification). The reproducibility and the reliability of the two systems were compared. In the study it was found that there were 14 cases that could not be classified by the Schatzker classification system. The three-column classification system demonstrated a higher interobserver reliability and can therefore be used as a supplement to the conventional Schatzker classification, especially in cases of complex fractures with posterior comminution. ${ }^{6}$

The final aspect of the study was to look at whether threedimensional computed tomography increased the reliability of classification systems for tibial plateau fractures. Hu concluded that three-dimensional computed tomography improved intra-observer and inter-observer reliability of classification systems for tibial plateau fractures when compared to plain radiographs and two-dimensional CT images. ${ }^{7}$
The aim of this study is to determine the ability of the Schatzker classification to predict the presence of posteromedial fragmentation in tibial plateau fractures and to determine the incidence of posteromedial fragmentation in the lower group (Schatzker type 1 and 2) as well as in the higher group (Schatzker type 3 to 6).

\section{Method}

The Research Ethics Committee of the Faculty of Health Sciences, University of Pretoria provided ethical clearance for this study (Protocol October/2016).

Two hundred patients treated at the Steve Biko Hospital Complex and Eugene Marais Hospital during the period January 2006 to December 2015 were identified for possible inclusion in the study. Digital radiographs and records of these cases were retrieved to confirm whether inclusion criteria were met. To be included in the study, patients had to be older than 18 years of age with a tibial plateau fracture and an adequate view of the affected knee on an antero-posterior radiograph. Moreover, a CT scan of the affected area was a necessity.

The researcher assigned a unique case number to each of the cases that met the inclusion criteria. Personal details were kept anonymous.

Three independent trauma orthopaedic surgeons reviewed the antero-posterior radiographs. They were requested to classify fractures according to the Schatzker classification. ${ }^{3}$

A radiologist examined the CT scans for posteromedial fragmentation and measured the fragment with the largest diameter in millimetres by means of a single measurement, using the most adequate CT view available. It should be noted that lateral radiographs were not used in this study as the Schatzker classification is a system based on antero-posterior view only. Identifying the origin of a posterior fragment on a lateral $\mathrm{X}$-ray is difficult as the fragment could be located medially or laterally. In such circumstances the ideal is rather to revert to CT scans to delineate the one from the other as was done in this study.

The statistical analysis was conducted with IBM SPSS Statistics for Windows, Version 23.0 Armonk, NY.IBM Corp.

The demographic traits of the sample were determined for descriptive purposes. Percentage distributions were calculated for the fractures according the Schatzker classification system according to the categorisation of the three independent orthopaedic surgeons. The kappa statistic ${ }^{8}$ was calculated to indicate the level of agreement between the categorisation of the three independent orthopaedic surgeons.

Posteromedial fragmentation prevalence and mean diameter (in millimetres) per Schatzker type were descriptively reported in relation to the corresponding Schatzker categorisations. The mean age for cases with and without posteromedial fragmentation was compared using an independent sample's t-test. One-way analysis of variance was used to compare the mean diameter of the posteromedial fragmentation (widest measurement) per Schatzker type.

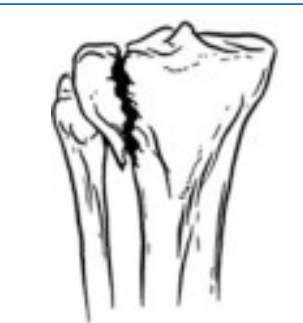

Type I
Split

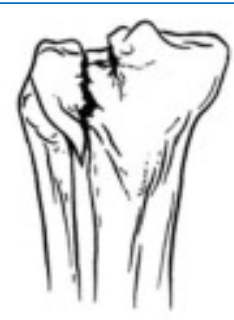

Type II

Split-depression

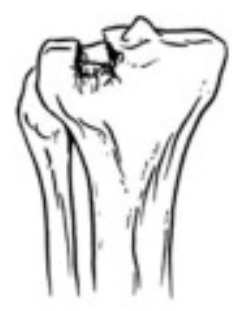

Type III

Central depression

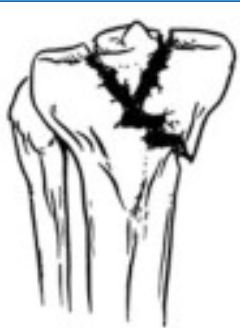

Type IV

Split fracture, medial plateau

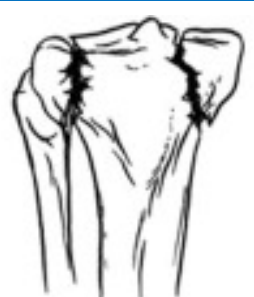

Type V

Bicondylar fracture

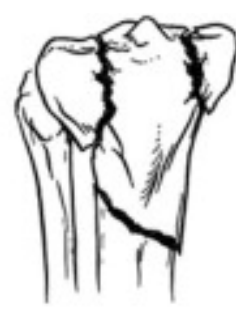

Type VI

Dissociation of

metaphysis and diaphysis 
Table I: Percentage distribution of fractures categorised according to the Schatzker classification system in relation to CT confirmation of posteromedial fragmentation

\begin{tabular}{|c|c|c|c|c|c|c|c|c|c|c|c|c|}
\hline \multirow{3}{*}{$\begin{array}{l}\text { Schatzker } \\
\text { classification }\end{array}$} & \multicolumn{4}{|c|}{ Orthopaedic surgeon 1} & \multicolumn{4}{|c|}{ Orthopaedic surgeon 2} & \multicolumn{4}{|c|}{ Orthopaedic surgeon 3} \\
\hline & \multicolumn{2}{|c|}{ All cases } & \multicolumn{2}{|c|}{$\begin{array}{l}\text { Posteromedial } \\
\text { fragmentation }\end{array}$} & \multicolumn{2}{|c|}{ All cases } & \multicolumn{2}{|c|}{$\begin{array}{l}\text { Posteromedial } \\
\text { fragmentation }\end{array}$} & \multicolumn{2}{|c|}{ All cases } & \multicolumn{2}{|c|}{$\begin{array}{l}\text { Posteromedial } \\
\text { fragmentation }\end{array}$} \\
\hline & $\mathrm{n}$ & $\%$ & $n$ & $\%$ & $\mathrm{n}$ & $\%$ & $n$ & $\%$ & $n$ & $\%$ & $n$ & $\%$ \\
\hline 1 & 11 & 16.4 & 1 & 5.3 & 9 & 13.4 & 1 & 5.3 & 10 & 14.9 & 1 & 5.3 \\
\hline 2 & 25 & 37.3 & 2 & 10.5 & 28 & 41.8 & 2 & 10.5 & 31 & 46.3 & 4 & 21.1 \\
\hline 3 & 4 & 6.0 & 1 & 5.3 & 6 & 9.0 & 2 & 10.5 & 4 & 6.0 & 1 & 5.3 \\
\hline 4 & 7 & 10.4 & 3 & 15.8 & 5 & 7.5 & 3 & 15.8 & 8 & 11.9 & 5 & 26.3 \\
\hline 5 & 7 & 10.4 & 5 & 26.3 & 2 & 3.0 & 2 & 10.5 & 7 & 10.4 & 3 & 15.8 \\
\hline 6 & 13 & 19.4 & 7 & 36.8 & 17 & 25.4 & 9 & 47.4 & 7 & 10.4 & 5 & 26.3 \\
\hline 1 and 2 subtotal & 36 & 53.7 & 3 & $15.8^{\star}$ & 37 & 55.2 & 3 & $15.8^{\star}$ & 41 & 61.2 & 5 & $26.3^{*}$ \\
\hline 3 to 6 subtotal & 31 & 46.3 & 16 & $84.2^{*}$ & 30 & 47.8 & 16 & $84.2^{\star}$ & 26 & 38.8 & 14 & $73.7^{*}$ \\
\hline Total & 67 & & 19 & & 67 & & 19 & & 67 & & 19 & \\
\hline
\end{tabular}

${ }^{*}$ chi $^{2}$ test: $P<0.001$

\section{Results}

Sixty-seven of the 200 identified hospital cases met the inclusion criteria, of which 44 were males and 33 females. The mean age of the included cases was 44.79 (standard deviation [SD]: 14.03, 95\% $\mathrm{Cl}$ (confidence interval: 41.37-48.21; range 24-93) years.

Table / shows the percentage distribution of fractures classified, according to the Schatzker classification system, by the three independent orthopaedic surgeons. All three surgeons classified the majority of fractures as either Schatzker type 1 or 2 . Type 1 and 2 fractures constituted the majority, ranging between between 53.70 and 61.20 for the three independent assessments. The inter-rater reliability test yielded kappa statistics of $0.49,0.51$ and 0.54 when comparing the agreement between orthopaedic surgeons 1 and 2, 1 and 3 , and 2 and 3 , respectively, which constituted 'weak' agreement ${ }^{9}$ between the surgeons for the above-mentioned classification.

According to the CT scan assessment, a posteromedial fragment was present in 19 cases.

The mean age where this occurred was 48.78 (SD: 17.33) years as opposed to the mean age of 43.21 (SD: 12.34) years for the cases where posteromedial fragmentation was not prevalent (independent samples t-test, $\mathrm{P}=0.143,95 \% \mathrm{Cl}$ of the difference: -13.11 to 1.95 years). Furthermore, $75 \%$ of the females in the sample presented with posteromedial fragmentation compared to $65.20 \%$ of the males (chi²-test, $\mathrm{P}=0.399$ ).

Table / also indicates the prevalence of posteromedial fragmentation in relation to the Schatzker types. According to the three independent assessments, posteromedial fragmentation occurred in $84.2 \%$, $84.2 \%$ and $73.7 \%$ of type 3 to 6 cases compared to $15.8 \%, 15.8 \%$ and $26.3 \%$ in type 1 and 2 cases. These differences were statistically significant (chi²-test: $\mathrm{P}<0.001)$.

The mean posteromedial fragmentation length was $45.22 \mathrm{~mm}$ (SD: 10.22; 95\% Cl: 40.29-50.15). The average size of the posteromedial fragment in males overall was 39.18 (SD: 10.78) mm and 49.65 (SD: 7.45$) \mathrm{mm}$ in females $(\mathrm{P}=0.022 ; 95 \% \mathrm{Cl}$ of the mean difference: -19.31 to -1.71$)$. The mean length of the posteromedial fragmentation ranged from 41.87 to $47.77 \mathrm{~mm}$ for Schatzker 1 and 2 fractures and 44.74 to $46.12 \mathrm{~mm}$ for Schatzker 3 to 6 for the three assessors (statistically not significant (Table II) [T-test, $\mathrm{P}=0.536$, $P=0.551$ and $P=0.652])$.

\section{Discussion}

The Schatzker classification is the most widely used and internationally known classification system for tibial plateau fractures. ${ }^{3}$ It has the best inter-observer reliability and is taught at most government institutions as the way to quantify the severity of these injuries. It is also mainly an anteroposterior view-based system whereby a lateral view plays no role in this classification system.

All tibial plateau fractures, however, cannot be reliably classified by using the Schatzker classification and hence the role of a CT scan in these fractures to delineate the extent is important. The use of a CT scan in all types of tibial plateau fractures is controversial as it is costly to scan every patient with this fracture. The association between posteromedial fragmentation and types 3 to 6 is known as all these fracture types are further investigated by a CT scan to delineate the fragmentation and configuration. Type 1 and 2 fractures in some institutions do not routinely get a CT scan and hence the exact incidence of posteromedial fragmentation is not known.

Overall, posteromedial fragmentation was more common in females than males in all categories, although not statistically significant. Based on the assessment of the three orthopaedic surgeons in this study it appears as if a substantial number $(15.8 \%-$ $26.3 \%$ ) of posteromedial fragmentation may be missed in the lower categories if a CT scan is not done. A range of $41.87-47.77 \mathrm{~mm}$ in the widest diameter of the fragment was noted in Schatzker 1 and Schatzker 2 fractures. There were no studies that evaluated this

Table II: Posteromedial fragmentation length differences according to a Schatzker classification dichotomy (Class 1 and 2 versus 3 to 6 )

\begin{tabular}{|c|c|c|c|c|c|c|c|c|c|}
\hline \multirow{3}{*}{ Schatzker classification } & \multicolumn{3}{|c|}{ Orthopaedic surgeon 1} & \multicolumn{3}{|c|}{ Orthopaedic surgeon 2} & \multicolumn{3}{|c|}{ Orthopaedic surgeon 3} \\
\hline & \multicolumn{3}{|c|}{ Mean $(\mathrm{X})$ fracture length $(\mathrm{mm})$} & \multicolumn{3}{|c|}{ Mean $(X)$ fracture length $(\mathrm{mm})$} & \multicolumn{3}{|c|}{ Mean $(\mathrm{X})$ fracture length $(\mathrm{mm})$} \\
\hline & $\mathbf{n}$ & $\mathbf{x}$ & SD & $\mathbf{n}$ & $\mathbf{x}$ & SD & $\mathbf{n}$ & $\mathbf{x}$ & SD \\
\hline 1 and 2 & 3 & 47.77 & 9.25 & 3 & 41.87 & 18.86 & 5 & 42.70 & 13.95 \\
\hline 3 to 6 & 16 & 44.74 & 10.60 & 16 & 45.85 & 8.67 & 14 & 46.12 & 9.03 \\
\hline $95 \% \mathrm{Cl}$ of the mean difference & \multicolumn{3}{|c|}{-10.86 to 16.90} & \multicolumn{3}{|c|}{-17.78 to 9.83} & \multicolumn{3}{|c|}{-14.85 to 8.01} \\
\hline$P$ & \multicolumn{3}{|c|}{0.652} & \multicolumn{3}{|c|}{0.551} & \multicolumn{3}{|c|}{0.536} \\
\hline
\end{tabular}


correlation and hence a clear comparison could not be made to compare to known literature. We found an incidence of posteromedial fragmentation in the higher categories (Schatzker 3 to 6) of between $73.7 \%-84.2 \%$ which is higher than reported in current literature where a percentage of between $59 \%$ and $74 \%$ is noted. This can be attributed to the small sample size of our study.

Cuellar et al. state that the posteromedial fragment in tibial plateau fractures is considered unstable and hence proceeded to provide biomechanical evidence in this regard. They reviewed the effect of the size of the posteromedial fragment and the effect on stability of the knee. They found that for fragments the size of $10 \mathrm{~mm}$, medial femoral condyle displacement was only unaffected when the knee was taken to a range of 30 degrees of flexion. Beyond this measurement there was medial femoral condyle displacement. Fragments larger than $20 \mathrm{~mm}$ added to medial femoral condyle displacement in all ranges of flexion from baseline to 90 degrees of flexion. This was a biomechanical study done in cadavers. During non-weightbearing knee range of motion exercises such as compression, flexion, torque and varus-valgus stress, the size of the posteromedial fragment is important and can lead to subsequent fracture instability. The correlation between the exact fragment size and need for fixation is unknown, but the biomechanical disadvantage of not addressing the fragment is present. ${ }^{9}$

The standard approach to the fixation of a tibial plateau fracture is most often laterally or medially depending on the fracture characteristics. If there is a significantly sized posteromedial fragment, a posterior approach is preferable to address and buttress the fragment from posterior. In this case a standard approach might not suffice to provide and maintain adequate alignment, reconstruct an anatomical joint surface and provide early range of motion. Yoram et al. described a posteromedial approach to address the posteromedial fragment, which is a variation of the conventional medial approach. The importance is that one needs to anticipate fixing the posteromedial fragment before using this approach. ${ }^{10}$

The limitation of our study is that it is a retrospective study with a small sample size of 67 patients. A larger sample size will contribute to better statistical significance with regard to the fracture lengths as well as inter-relater correlation.

\section{Conclusion}

The Schatzker classification by itself is not adequate to identify all fractures with posteromedial fragmentation. There is a higher incidence of posteromedial fragmentation with fracture types 3 to 6 tibial plateau fractures. There is a high probability of missing a significantly sized posteromedial fragment in Schatzker type 1 and 2 fractures that might influence and compromise fracture stability, joint congruency and the ability to rehabilitate optimally.

\section{Ethics statement}

The Research Ethics Committee of the Faculty of Health Sciences, University of Pretoria, provided ethical clearance for this study (Protocol October/2016).

\section{References}

1. Cole P, Levy B, Schatzker J, Watson JT. Tibial plateau fractures. Skeletal Trauma: Basic Science Management and Reconstruction. Philadelphia: Saunders Elsevier. 2009;2201-87

2. Bare DP, Nork SE, Mills WJ, Coles CP, Henley MB, Benirschke SK Functional outcomes of severe bicondylar tibial plateu fractures treated with dual incisions and medial and lateral plates. J Bone Joint Surg. 2006 Aug;88(8):1713-21.

3. David W, Zeltser MD, Seth S, Leopold MD. Classification in brief Schatzker classification of tibial plateau fractures. Clin Orthop Relat Res. 2013 Feb:471(2):371-74.
4. David P, Barei TJ, O'Mara LA, Taitsman RP, Dunbar SE. Frequency and fracture morphology of the posteromedial fragment in bicondylar tibial plateau fracture patterns. J Orthop Trauma. 2008 April;22(4):176-82.

5. Martijn AJ, te Stroet HM, Biert J, van Kampoen A. The value of CT scan compared to plain radiographs for the classification and treatment plan in tibial plateau fractures. Emergency Radiology. 2011 Aug;18(4):279-83.

6. Zhu Y, Yang G, Luo C-F, Smith WR, Hu C-F, Gao H, Zhong B, Zeng B-F. Computed tomography-based three column classification in tibial plateau fractures: Introduction of its utility and assessment of its reproducibility. J Trauma Acute Care Surg. 2012 Dec;73(6):731-37.

7. Hu Y, Ye F-G, Ji A-Y, Qiao G-X, Liu H-F. Three-dimensional computed tomography imaging increases the reliability of classification systems for tibial plateau fractures. Injury. 2009 Dec;40(12):1282-85.

8. McHugh ML. Interrelated reliability. The kappa statistic. Biochem Med 2012 Oct;22(3):276-82.

9. Ceullar VG, Martinez D, Immerman I. A biomechanical study of posteromedial tibial plateau fracture stability: Do they all require fixation? J Orthop Trauma 2015 July;29(2):325-30.

10. Yoram A, Gardner MJ, Helfet DL. Posteromedial supine approach for reduction and fixation of medial and bicondylar tibial plateau fractures. J Orthop Trauma 2008 May;22(5):357-62. 